\title{
Just the facts: diagnosis and management of intimate partner violence in the emergency department
}

\author{
Megan Brenkel ${ }^{1} \cdot$ Alexandre Coutin $^{1,2} \cdot$ Shahbaz Syed $^{1,2} \cdot$ Kari Sampsel $^{1,2}$ (I)
}

Received: 29 September 2021 / Accepted: 5 October 2021 / Published online: 15 October 2021

(C) The Author(s), under exclusive licence to Canadian Association of Emergency Physicians (CAEP)/ Association Canadienne de Médecine d'Urgence (ACMU) 2021

\section{Clinical scenario}

A 36-year-old female presents to the emergency department (ED) with a diffuse headache. She has previously visited the ED three times for the same presentation, and had normal investigations, including a CT head. On assessment, she appears withdrawn and makes poor eye contact. When you examine her, you notice a bruise behind her right ear and along the posterior angle of her jaw. When you ask her about them, she looks away and does not provide a response.

IPV or Intimate Partner Violence-describes physical violence, sexual violence, stalking, or psychological harm by a current or former partner or spouse. This type of violence can occur among heterosexual or same-sex couples and does not require sexual intimacy [1].

\section{Should we screen for intimate partner violence? What factors should increase my suspicion of intimate partner violence?}

Intimate partner violence exists across all age spans, ethnicity, and socioeconomic backgrounds, affecting 1 in 6 women and 1 in 9 men in Canada [2]. Emergency physicians have a unique opportunity to identify victims of intimate partner violence and should be on high alert for suspicious presentations. This includes any vague/changing history, delays in seeking care, statements that patient is 'accident prone', traumatic injury, frequent ED visits for injuries, chronic pain or other somatic complaints, unintended pregnancy, and mental health concerns (including depression, suicidal ideation/attempts, and evidence of substance/alcohol misuse).

Kari Sampsel

ksampsel@toh.ca

University of Ottawa, Ottawa, ON, Canada

2 Department of Emergency Medicine, The Ottawa Hospital, 1053 Carling Avenue, Ottawa, ON K1Y 4A9, Canada
It is also important to recognize an inappropriate affect, overly attentive partner, and reluctance or difficulty with physical examination. Injury patterns that are inconsistent with the provided history should raise concern. Injuries that are centrally located (i.e., trunk, chest, genitals), bilateral (i.e., both arms or legs), defensive (i.e., bruises or fractures to the hands or forearms), or involving the head, neck, and face should also be considered. Lastly, remember to be on the lookout for injury findings in concealed areas, and/or at various stages of healing $[3,4]$.

\section{How do I ask about intimate partner violence? How do I respond to a disclosure? What should I avoid asking?}

While many screening tools are cumbersome and difficult to use in a fast-paced ED setting, there are two general questions that are efficient and effective: (1) "Are you safe at home?" And (2) "Has someone hurt you?" Accessible and broad language avoids labelling survivors as abused. Many survivors may not see themselves this way. Furthermore, studies show that survivors do not mind these questions and encourage them $[5,6]$.

It is crucial to respond to the disclosure of intimate partner violence in a supportive manner that indicates that you believe the patient. On average, it takes eight visits to healthcare providers before a disclosure is made [6]. A respectful, validating response to disclosure results in sixfold reduction in mental health sequelae, substance misuse, and PTSD [6].

It is imperative that clinicians avoid questions like "why didn't you leave?", "what did you do to set them off?", or "what were you wearing?". These statements are wholly unhelpful, and can disrupt the physician-patient relationship, thereby jeopardizing the patient's healthcare experience [6]. 


\section{What are the key components of intimate partner violence injury assessment and treatment?}

As with any other traumatic injury, one's assessment should be guided by the history. The examination must be done sensitively, which includes frequently checking in with the patient. Avoid surprises by ensuring the patient understands what you are going to examine next. If an injury is confined to one location, a head-to-toe exam may not be warranted.

Similarly, treatment is guided by the injury. It may include wound care, analgesia, splinting, or casting. Medical treatment always comes before forensic evidence collection.

\section{How do I document assessments on intimate partner violence patients?}

The patient's medical records may be summoned to court, which reinforces the need for clear and legible documentation. A helpful approach is to consider how one would document a bar fight. Consider what details of the assault you would include. Where/how/how many times/with what was the survivor assaulted with? Did they lose consciousness? Were they strangled? Who was the assailant and what is their relationship to the patient? Wherever possible, include patient statements with quotations. Comprehensive documentation of the physical exam includes a mental status exam describing the patient's demeanor and appearance. Document old and new injuries and include the size, type, and location. It may be helpful to include a body map of the injuries, and/or with the patient's consent, photographs of the injuries (completed by the specialized treatment service). Including details such as what the assailant was wearing is unnecessary, as it has no bearing on your medical care. Record a safety assessment, specialist referral(s), and disposition plan. Finally, label the discharge diagnosis as intimate partner violence. Do not use the word "alleged" - this is a legal term and has no place in Medicine. We would not document alleged MI; therefore, we should not use this term here.

\section{How do l ensure my patient is safe?}

To ensure safety, the patient should not be left in the waiting room. Instead, use a visible location within the ED that is inaccessible to their assailant. Remember, the highest risk for a survivor is when he or she leaves or seeks care. There is a $4 \times$ increased likelihood of being murdered [7].

Furthermore, a safety plan for discharge is necessary. This includes: Where are they going? How are they going to get there? Does their assailant have access to them there?

It is important to recognize that most victims will return to their assailant, and physicians must avoid responses of disbelief and disapproval [6]. Safety planning when patients are in the precontemplation/contemplation stage may include having a protected room with an escape for outbursts, having a 'go-bag' with essential documents, using code words with friends and/or family and inputting resources into their phone [8]. Given the complexity and importance of individualized safety planning, if available, involve specialty intimate partner violence services or Social Work for assistance with this. Remember to exercise caution in providing patients with written materials as these can trigger further violence towards the patient once they are discharged.

A woman is murdered every 6 days in Canada [7], with indigenous women, transgender women of colour, and those participating in sex work being at disproportionately high risk for violence $[9,10]$.

\section{How/who do I refer for further care and support?}

Specialized treatment services are available for additional support. These services provide crisis intervention and support, documentation of assault, assessment and documentation of injuries (including photographs), testing and treatment for STIs, forensic evidence collection, safety planning, and referrals to community agencies for support. They also provide non-acute care directly and follow-up care. It is important to offer this support to all intimate partner violence patients. Remind the patient that these services are not an extension of the police, however, they can involve them if requested. Specialized services can be accessed in the following ways:

- In Ontario: all hospitals have a referral relationship with a specialized treatment service. To find one: https://www. sadvtreatmentcentres.ca/find-a-centre/

- Across Canada/Internationally: https://www.forensicnu rses.org/

- Google intimate partner violence treatment centre in your area

\section{Case resolution}

After emphasizing that she is safe, you ask your patient directly about intimate partner violence. She discloses that she is being abused by her live-in partner and starts to cry. You respond in a supportive manner, validate her decision to seek help in the ED, and assess her safety. On exam, there are no injuries that require imaging or treatment. You then offer to have the patient seen by a specialized intimate partner violence program, who may assist with safety planning and referrals for community support. You provide analgesia for her headache and keep her within the department until she is seen by the specialist. You document the discharge diagnosis as intimate partner violence. 


\section{JUST THE FACTS - IPV \\ - Physical, sexual, or psychological violence by a current or former partner or spouse \\ - Can affect ANYONE. Those who are younger, indigenous, transgender, \\ pregnant, disabled, racialized, impoverished, or involved in sex work are at \\ increased risk}

\section{A woman is murdered every 6 d d y $\mathbf{S}$ in canada}

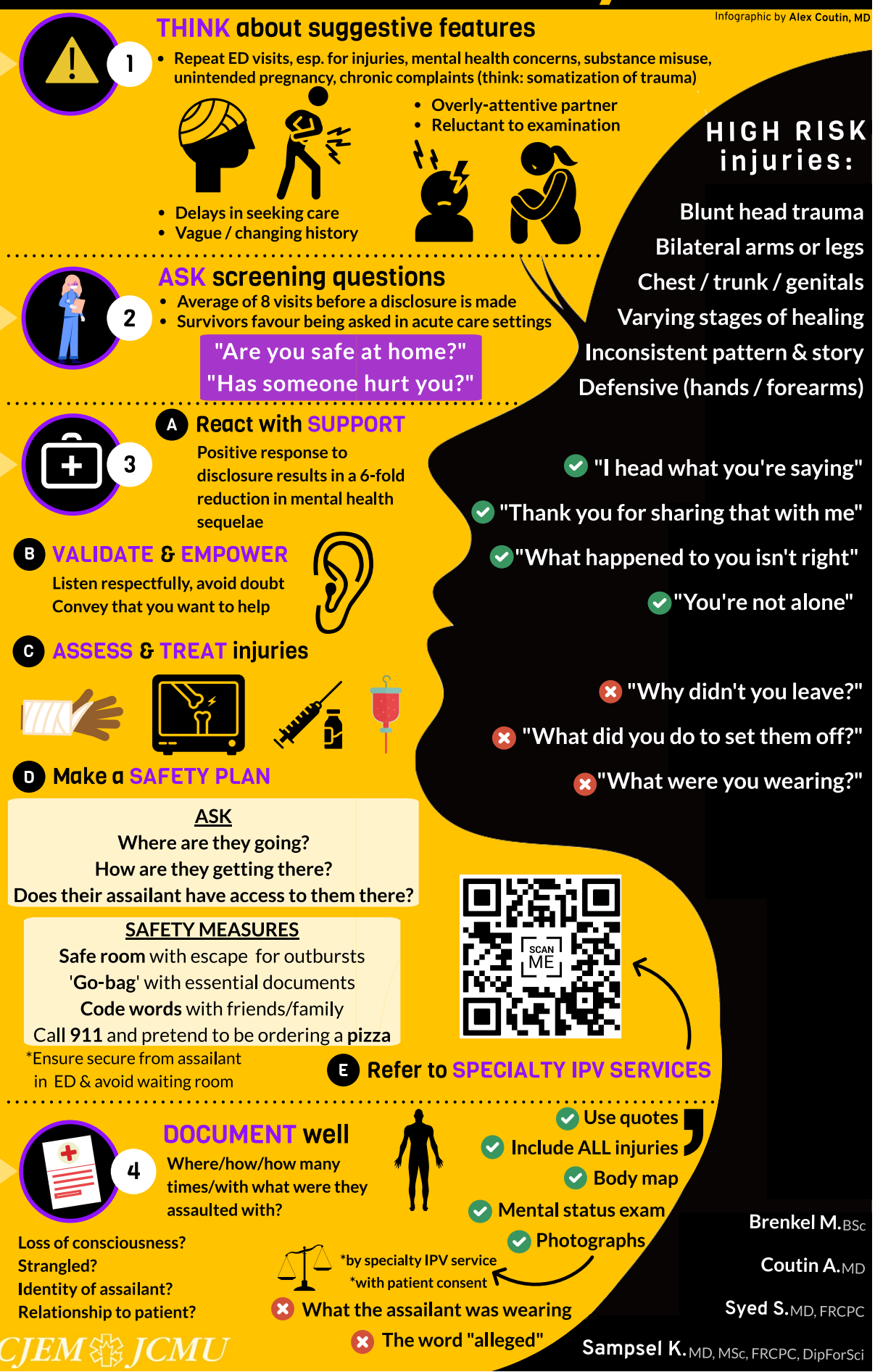




\section{Declarations}

Conflicts of interest There are no conflicts of interest to declare.

\section{References}

1. https://www.cdc.gov/violenceprevention/intimatepartnerviolence/ index.html; Accessed 26 Aug 2021.

2. Statistics Canada. Family violence: How big is the problem in Canada? 2018. https://www.canada.ca/en/public-health/servi ces/health-promotion/stop-family-violence/problem-canada.html Accessed 26 Aug 2021.

3. Sampsel K. Chapter 99-intimate partner violence emergency medicine secrets. 7th ed. New York: Elsevier; 2020.

4. Walls R, Hockberger R, Gausche-Hill MM. Intimate partner violence and abuse. Rosen's emergency medicine-concepts and clinical practice e-book. New York: Elsevier Health Sciences; 2017.

5. Ansara DL, Hindin MJ. Psychosocial consequences of intimate partner violence for women and men in Canada. J Interpers
Violence. 2011;26(8):1628-45. https://doi.org/10.1177/08862 60510370600

6. Ansara DL, Hindin MJ. Formal and informal help-seeking associated with women's and men's experiences of intimate partner violence in Canada. Soc Sci Med. 2010;70(7):1011-8. https://doi. org/10.1016/j.socscimed.2009.12.009.

7. Statistics Canada Incident-based crime statistics, by detailed violation. http://www23.statcan.gc.ca/imdb/p2SV.pl?Function= getSurvey\&lang $=e n \& d b=i m d b \& a d m=8 \&$ dis $=2 \& S D D S=3302$. Published 2016. Accessed 27 Aug 2021.

8. Choo EK, Houry DE. Managing intimate partner violence in the emergency department. Ann Emerg Med. 2015;65(4):447-51.

9. Joseph J. Transphobic femicide: an intersectional perspective. In: Joseph J, Jergenson S, editors. An international perspective on contemporary developments in victimology. Cham: Springer; 2020.

10. Wathen CN, MacGregor JCD, Tanaka M, et al. The impact of intimate partner violence on the health and work of gender and sexual minorities in Canada. Int J Public Health. 2018;63:945-55. https://doi.org/10.1007/s00038-018-1127-1. 